\title{
Cognitive decline and mortality in a community- based sample of homeless and precariously housed adults: 9-year prospective study
}

Kristina M. Gicas, Andrea A. Jones, Allen E. Thornton, Anna Petersson, Emily Livingston, Kristina Waclawik, William J. Panenka, Alasdair M. Barr, Donna J. Lang, Fidel Vila-Rodriguez, Olga Leonova, Ric M. Procyshyn, Tari Buchanan, G. William MacEwan and William G. Honer

\section{Background}

Homeless and precariously housed individuals experience a high burden of comorbid illnesses, and excess mortality. Cross-sectional studies report a high rate of cognitive impairment. Longterm trajectories have not been well investigated in this group.

\section{Aims}

To longitudinally assess risks for premature and/or accelerated cognitive ageing, and the relationship with early mortality in homeless and precariously housed people.

\section{Method}

This is a 9-year community-based study of 375 homeless and precariously housed individuals from Vancouver, Canada. Annual cognitive testing assessed verbal learning and memory, and inhibitory control. Linear mixed-effects models examined associations between clinical risk factors (traumatic brain injury, psychotic disorders, viral exposure, alcohol dependence) and cognitive change over 9 years. Cox regression models examined the association between cognition and mortality.

\section{Results}

Traumatic brain injury and alcohol dependence were associated with decline in verbal memory. Inhibitory control declined, independent of risk factors and to a greater extent in those who died during the study. Better inhibitory control was associated with a $6.6 \%$ lower risk of mortality at study entry, with a $0.3 \%$ greater effect for each year of life. For each one-point increase in the Charlson Comorbidity Index score at study entry, the risk of mortality was $9.9 \%$ higher, and was consistent across age. Adjusting for comorbidities, inhibitory control remained a significant predictor of mortality.

\section{Conclusions}

Findings raise the possibility of a premature onset, and accelerated trajectory, of cognitive ageing in this group of homeless and precariously housed people. Traumatic brain injury, alcohol dependence and cognition could be treatment priorities.

\section{Declaration of interest}

A.E.T. reports grants from Canadian Institute for Health Research, William and Ada Isabelle Steel Fund, Simon Fraser University Psychology Department. W.J.P. reports personal fees from Abbatis Bioceuticals, Medipure Pharmaceuticals and is owner of Translational Life Sciences. F.V.-R. reports personal fees from Janssen; and grants from Canadian Institutes of Health Research, Michael Smith Foundation for Health Research, Vancouver Coastal Health Research Institute, and Brain Canada. R.M.P. reports personal fees from Janssen, Lundbeck and Otsuka. W.G.H. reports personal fees from Canadian Agency for Drugs and Technology in Health, AlphaSights, Guidepoint, Translational Life Sciences, Otsuka, Lundbeck, and Newron; grants from Canadian Institutes of Health Research, BC Mental Health and Addictions Services; and has been a consultant (nonpaid) for In Silico. K.M.G, A.M.B., D.J.L., O.L., A.A.J, A.P., E.L., K.W., T.B., G.W.M. report no competing interests.

\section{Keywords}

Mortality; comorbidity; cognition; marginalisation; ageing.

\section{Copyright and usage}

(C) The Author(s) 2020. This is an Open Access article, distributed under the terms of the Creative Commons AttributionNonCommercial-NoDerivatives licence (http://creativecommons.org/licenses/by-nc-nd/4.0/, which permits non-commercial re-use, distribution, and reproduction in any medium, provided the original work is unaltered and is properly cited. The written permission of Cambridge University Press must be obtained for commercial re-use or in order to create a derivative work.
Social marginalisation in high-income countries is increasingly recognised as a risk factor for poor health, a high burden of multimorbidity and premature mortality. ${ }^{1}$ Homeless people represent a population vulnerable to the 'tri- morbidity' of social marginalisation: mental illness, addiction and physical illness. ${ }^{2}$ The health risks and consequences of living in precarious housing or absolute homelessness include high rates of psychotic disorders (up to $42 \%$ ), substance use disorders (up to $54 \%$ ), traumatic brain injury (up to 53\%) and HIV infection (up to $21 \%$ ). ${ }^{3}$ These illnesses contribute to excess mortality, with risk of all-cause mortality estimated to be 4.83-11.60 times higher than expected. ${ }^{1}$ Cross-sectional studies indicate multimorbid illness is associated with functional impairment in youth and older homeless or precariously housed people, however, the long-term trajectories of functioning are not well described. ${ }^{4,5}$ Age-related functional impairments are disproportionately higher in homeless people over 50 years old, possibly creating vulnerability to early dementia and accelerated ageing. ${ }^{6,7}$ Cognitive functioning is of particular interest given its importance for daily living, yet it is an often overlooked health outcome. Cross-sectional studies report global cognitive impairment in approximately $25 \%$ of homeless people, ${ }^{8}$ with selective deficits in attention, executive functioning and most prominently, verbal memory. ${ }^{6,7,9,10}$ Cognitive dysfunction occurs across the age spectrum, including in homeless youth. ${ }^{11,12}$ However, whether impairments are static, reversible or progressive is unclear.

Risk factors for cognitive dysfunction are not well delineated among marginalised people, but generally a multifactorial aetiology is presumed. ${ }^{8,10}$ Illnesses associated with premature or accelerated ageing, and found with greater prevalence in marginalised people, include schizophrenia, traumatic brain injury, alcohol dependence and HIV infection. ${ }^{13-16}$ Independent of age-related illnesses, cognitive dysfunction may be a critical marker of diminishing robustness 
in biological systems associated with premature mortality. ${ }^{17,18}$ Identifying vulnerabilities for poor long-term outcomes in homeless people is essential to establish priorities for early interventions.

\section{Aims and hypotheses}

To the best of our knowledge, cognition has never been examined longitudinally in a homeless or precariously housed sample. The current study involves a comprehensive 9-year prospective evaluation of over 350 people who are living in such a situation in a large urban center. ${ }^{4}$ The objectives were: (a) to describe trajectories of change in cognition, including verbal learning and memory, and inhibitory control; (b) to evaluate risk factors for cognitive change; and (c) to examine whether cognition is a predictor of mortality independent of chronic medical comorbidities. Older age and select risk factors (psychosis, alcohol use, traumatic brain injury, viral infection burden) are hypothesised to be associated with steeper rates of cognitive decline, and in turn, a greater risk of mortality. Our decision to focus on verbal learning, memory, and inhibitory control as our cognitive outcomes is reflected in the sensitivity of these measures to disruption from psychiatric and neurological illnesses, and the translational value given the tests we use are routinely administered in clinical neuropsychological assessments.

\section{Method}

\section{Study design and participants}

Participants were recruited from single-room occupancy hotels $(n=310)$ and a downtown community courthouse $(n=65)$ located within a neighbourhood described as Canada's 'poorest postal code' ${ }^{19,20}$ This single-room occupancy hotel accommodation meets a Canadian definition of 'precarious housing'; falling below standards of adequacy, affordability or suitability and creating risk for homelessness. ${ }^{21}$ People who were 18 years or older and English-speaking were eligible. Written informed consent was obtained from all participants. A full description of the nature and purpose of the study was provided to all participants with a minimum of $24 \mathrm{~h}$ to consider this information prior to enrolment. A small monetary honorarium was provided for each completed assessment.

The authors assert that all procedures contributing to this work comply with the ethical standards of the relevant national and institutional committees on human experimentation and with the Helsinki Declaration of 1975, as revised in 2008. All procedures involving human participants were approved by Clinical Research Ethics Board of the University of British Columbia and the Simon Fraser University Office of Research Ethics (H08-00521).

\section{Cognitive measures}

Cognitive tests were administered by research assistants, supervised by a neuropsychologist (A.E.T.), at baseline and annually thereafter. Verbal learning was assessed with the total immediate recall score and verbal memory with the total delayed recall score from the Hopkins Verbal Learning Test-Revised (HVLT-R). ${ }^{22}$ Alternate forms were counterbalanced in the first 2 years (Forms 1 and 2) and then consecutively administered (Forms 3-6), re-starting with Form 1 at the seventh test administration. Inhibitory control, a component process of executive functioning, was assessed with the total correct score on the Stroop colour-word subtest. Mean demographically corrected $T$-scores (population mean 50 , s.d. $=10$ ) for each cognitive measure were derived from normative tables provided in the respective test manuals, providing a benchmark for expected level of cognitive performance in the absence of central nervous system dysfunction and facilitating comparison with other studies. Examiners rated the validity of each test score from 1, clearly invalid to 5 , clearly valid. Tests scores rated as a 3 (questionably valid) or lower were considered invalid. This enables us to identify cognitive data that may have been invalidated by extraneous factors such as acute psychotic episodes, intoxication and extreme participant fatigue. Additional details are described elsewhere. ${ }^{10}$

\section{Clinical measures}

Clinical data were collected at enrolment by psychiatrists and research assistants independent of cognitive testing. Using information obtained from an interview with a psychiatrist (including mental status and neurological examinations), a Mini-International Neuropsychiatric Interview ${ }^{23}$ and a review of medical records, all available data were used to make comprehensive psychiatric diagnoses according to DSM-IV-TR criteria. ${ }^{24}$ Psychotic disorders included schizophrenia, schizoaffective disorder, psychosis not otherwise specified, substance-induced psychosis, mood disorder with psychotic features, or delusional disorder. ${ }^{25}$ A diagnosis of clinical cognitive impairment was made by a psychiatrist (W.G.H.) according to DSM-IV-TR criteria for dementia, amnestic disorder, and cognitive disorder not otherwise specified (as per DSM-IV Appendix B research criteria for mild cognitive impairment) using information from participant histories, cognitive testing and neurological examination. For descriptive and statistical analyses, we used a $T$-score of less than or equal to 1.5 s.d. below the normative mean to define scores that fall within the clinically impaired range on a given test, in accord with existing guidelines ${ }^{26}$ and with other studies in homeless people. , $12^{2}$

A history of traumatic brain injury was ascertained using a structured medical history questionnaire and defined as the occurrence of at least one injury to the head associated with any duration of loss of consciousness, confusion and/or memory loss, or neuroradiological evidence of trauma on whole brain images collected on a 3T Phillips Achieva scanner, or evidence of persistent sequelae attributable to injury (for example seizures, need for anticonvulsants, organic personality disorder). We adopted an inclusive definition of traumatic brain injury by grouping individuals as either having a history based on the above criteria, or no history, a previously used approach. ${ }^{27}$ Blood samples were drawn and submitted for HIV, hepatitis $\mathrm{B}$, hepatitis $\mathrm{C}(\mathrm{HCV})$, herpes simplex and cytomegalovirus serologies. Total virus exposure burden was quantified by summing seropositive results across the five viruses. ${ }^{28}$ Quantitative polymerase chain reaction ( $\mathrm{qPCR}$ ) determined the presence of an active infection for participants who were HCV seropositive. The Charlson Comorbidity Index was used to quantify cooccurring medical conditions according to the Charlson weighting scheme and adding one point for each decade of life over 40 years. ${ }^{29}$ Sociodemographic data was collected by research assistants using structured interviews. Duration of years living in homelessness or marginal housing within the neighbourhood was calculated as the most recent, relatively continuous period up to study entry. Mortality during the study was confirmed with Coroner's reports and hospital records.

\section{Statistical analysis}

\section{Cognition as a longitudinal outcome}

A series of linear mixed-effects models for longitudinal data were constructed for the three cognitive outcomes (verbal learning, verbal memory, inhibitory control). Random intercepts were included to account for between-subject variability and random slopes for time to account for repeated measures within subjects. Final models were determined by iteratively adding and removing 
fixed and random effects and comparing models using the Akaike Information Criterion and log likelihood ratio tests where appropriate. Age, gender and education were included as covariates. Timeinvariant, primary risk factors (history of traumatic brain injury, alcohol dependence, psychotic disorder, total virus exposure) were added as fixed effects to the covariate model. Significant interaction terms (risk factor $\times$ time $^{2}$ ) assessed by the log likelihood ratio test $(P<0.05)$ were retained for inclusion in the final model. Mortality during the study was included as a fixed effect to mitigate a healthy survivor bias. Additional interactions between time and covariates were explored. The Pseudo- $R^{2}$ statistic was used to quantify the proportion of explained variance in the random effects, with effect sizes defined by Cohen's guidelines for squared multiple correlation change (i.e. 0.02 , small; 0.13 , medium; and 0.26 , large) ${ }^{30}$ Checking of model assumptions, management of missing data, and sensitivity analyses are described in supplementary Appendix 1, available at https://doi.org/10.1192/bjo.2020.3.

Analyses of secondary risk factors were conducted for schizophrenia/schizoaffective disorder, substance-induced psychosis, HIV infection, HCV qPCR positive status, substance dependence diagnoses (stimulant, opioid, cannabis), and the Charlson Comorbidity Index. Duration (in years) living in the neighbourhood was used as a proxy for exposure to environmental impoverishment. Risk factors and their interactions were tested in separate models adjusted for demographic variables. Statistical analyses were performed in R (R Core Team, 2018) using the lme4 package. ${ }^{31}$

\section{Cognition as a predictor of mortality}

Survival analyses were conducted with left-truncated extended Cox regression models using age as the timescale to examine the association between cognition and risk of mortality. Participants who were lost to follow-up or who completed the study were right censored. Separate unadjusted regression models were conducted for the three cognitive variables, with cognitive scores entered as time-dependent variables. A test of the Schoenfeld residuals, and visual inspection of plots were used to assess proportionality. A time-varying coefficient was constructed by modelling the interaction between cognition and age. Significant interactions in the model suggest that the effect of cognition on risk of mortality varies by age, where the coefficient of the interaction term indicates the directional change in magnitude of the main effect for each additional year of life. Final adjusted models included the Charlson Comorbidity Index to control for the effect of co-occurring chronic physical illnesses on survival outcomes. Additional Cox regression analyses were conducted using clinical cognitive impairment status at study entry. Schoenfeld residuals were examined and age cut-points were considered where non-proportionality was evident. Analyses were conducted with R (R Core Team, 2018) using survival and survminer packages. ${ }^{32}$

\section{Results}

\section{Participant characteristics}

Data were collected between 17 November 2008 and 22 August 2018. Of approximately 515 potential participants, 375 (72.8\%) joined the study and 354 had completed a baseline cognitive assessment (see supplementary Fig. 1 for participant flow). Participant characteristics and risk factors for cognitive impairment are detailed in Table 1. The sociodemographic and clinical characteristics of our sample are comparable with other major Canadian studies of homeless people (see supplementary Table 1) and appear consistent with studies conducted in other high-income nations. ${ }^{3}$ This provides

\begin{tabular}{|c|c|c|}
\hline Characteristic or risk factor & Value & Total $n$ \\
\hline Gender, male: $n(\%)$ & $275(77.7)$ & 354 \\
\hline \multicolumn{3}{|l|}{ Ethnicity, $n(\%)$} \\
\hline White & $216(61.0)$ & 354 \\
\hline Indigenous & $97(27.4)$ & 354 \\
\hline Other/mixed & $41(11.6)$ & 354 \\
\hline Any formal employment, $n$ (\%) & $44(12.4)$ & 354 \\
\hline Ever homeless, $n(\%)$ & $233(65.8)$ & 354 \\
\hline Age, years: median (range) & $44(23-68)$ & 354 \\
\hline Education, years: median (range) & $10(2-16)$ & 354 \\
\hline Monthly income (Canadian dollars), median (range) & $826(185-3600)$ & 354 \\
\hline Estimated premorbid full-scale IQ, median (range) & $96(74-122)$ & 354 \\
\hline Duration in neighbourhood, years: median (range) & $7.2(0-47.6)$ & 354 \\
\hline Charlson Comorbidity Index, median (range) & $3(0-12)$ & 354 \\
\hline Any psychotic disorder $n(\%)$ & $169(47.7)$ & 354 \\
\hline Schizophrenia/schizoaffective disorder, $n$ (\%) & $48(13.6)$ & 354 \\
\hline Substance-induced psychotic disorder, $n$ (\%) & $62(17.5)$ & 354 \\
\hline Alcohol dependence, $n$ (\%) & $61(17.2)$ & 354 \\
\hline Cannabis dependence, $n$ (\%) & $111(31.4)$ & 354 \\
\hline Stimulant dependence, ${ }^{a} n(\%)$ & $293(82.8)$ & 354 \\
\hline Opioid dependence, $n(\%)$ & $150(42.5)$ & 353 \\
\hline Virus exposure burden, median (range) & $3(0-5)$ & 335 \\
\hline HIV seropositive, $n$ (\%) & $59(17.3)$ & 341 \\
\hline HCV seropositive, $n$ (\%) & $231(68.1)$ & 339 \\
\hline Clinical cognitive impairment, $n(\%)$ & $31(8.8)$ & 354 \\
\hline Dementia, $n$ (\%) & $6(1.7)$ & 354 \\
\hline Amnestic disorder, $n(\%)$ & $2(0.6)$ & 354 \\
\hline Cognitive disorder NOS, $n(\%)$ & $23(6.5)$ & 354 \\
\hline History of traumatic brain injury, $n(\%)$ & $174(49.2)$ & 354 \\
\hline MRI evidence or persistent sequelae, $n(\%)$ & $37(10.5)$ & 354 \\
\hline Hospital admission, $n(\%)$ & $144(40.7)$ & 354 \\
\hline
\end{tabular}


Table 2 Mixed-effects models of cognitive change over time and associations with risk factors ${ }^{a}$

\begin{tabular}{|c|c|c|c|c|c|c|c|c|c|}
\hline \multirow[b]{2}{*}{ Variable } & \multicolumn{3}{|c|}{ Verbal learning ${ }^{\mathrm{b}}$} & \multicolumn{3}{|c|}{ Verbal memoryc } & \multicolumn{3}{|c|}{ Inhibitory control $^{d}$} \\
\hline & Estimate & s.e. & $P$ & Estimate & s.e. & $P$ & Estimate & s.e. & $P$ \\
\hline Intercept & 20.13 & 0.57 & $<0.001$ & 6.41 & 0.29 & $<0.001$ & 35.26 & 1.05 & $<0.001$ \\
\hline Time (years) & 0.37 & 0.13 & 0.006 & 0.16 & 0.07 & 0.02 & 0.86 & 0.20 & $<0.001$ \\
\hline Time $^{2}$ (years) & -0.03 & 0.02 & 0.07 & -0.01 & 0.01 & 0.45 & -0.07 & 0.03 & 0.008 \\
\hline Gender (female) & 1.83 & 0.73 & 0.01 & 0.73 & 0.37 & $<0.05$ & 2.10 & 1.34 & 0.12 \\
\hline Age & -0.08 & 0.03 & 0.03 & -0.03 & 0.02 & 0.07 & -0.25 & 0.06 & $<0.001$ \\
\hline Education & 0.39 & 0.18 & 0.002 & 0.15 & 0.06 & 0.02 & 0.82 & 0.23 & 0.001 \\
\hline Died in study & -0.57 & 0.77 & 0.46 & -0.22 & 0.39 & 0.57 & -2.56 & 1.44 & 0.08 \\
\hline Died in study $\times$ time ${ }^{2}$ & - & - & - & -0.02 & 0.018 & 0.11 & -0.11 & 0.03 & 0.002 \\
\hline History of TBI & -0.71 & 0.59 & 0.23 & -0.11 & 0.30 & 0.70 & 1.01 & 1.08 & 0.35 \\
\hline History of TBI $\times$ time $^{2}$ & - & - & - & -0.02 & 0.01 & 0.007 & -0.03 & 0.02 & 0.07 \\
\hline Alcohol dependence & -1.44 & 0.74 & 0.05 & -0.09 & 0.38 & 0.82 & -1.02 & 1.39 & 0.46 \\
\hline Alcohol dependence $\times$ time $^{2}$ & - & - & - & -0.02 & 0.01 & 0.006 & - & - & - \\
\hline Total virus exposure burden & -0.69 & 0.25 & 0.006 & -0.32 & 0.12 & 0.01 & 0.34 & 0.46 & 0.46 \\
\hline Psychotic disorder & -1.58 & 0.58 & 0.006 & -0.72 & 0.29 & 0.01 & -2.06 & 1.06 & 0.05 \\
\hline \multicolumn{10}{|c|}{$\begin{array}{l}\text { TBI, traumatic brain injury; ' }- \text { indicates that the interaction was not included in the final adjusted model. } \\
\text { a. Models fit using restricted maximum likelihood estimation. } \text {. Time }^{2} \text { is a quadratic function (time } \times \text { time). } \\
\text { b. } n=295 \text {. Observations }=1670 \text {. } \\
\text { c. } n=294 \text {. Observations }=1634 \text {. } \\
\text { d. } n=289 \text {. Observations }=1606 \text {. }\end{array}$} \\
\hline
\end{tabular}

support for the possible relevance of our sample to regional and international contexts.

At baseline evaluation, the mean age-adjusted $T$-score for verbal learning was 30.5 (s.d. $=10.2$ ), falling in the clinically impaired range, with $68.1 \%$ of the participants scoring at or below the 1.5 s.d. cut-off used to define impairment on a given test. The mean end-point verbal learning $T$-score remained within the clinically impaired range (mean 33.6 , s.d. $=12.7$ ), with $57.6 \%$ of the participants scoring at or below the cut-off. For verbal memory, the mean baseline $\mathrm{T}$-score was also in the clinically impaired range (mean 31.5. s.d. $=11.0$ ), with $62.9 \%$ of participants scoring at or below the cut-off. The mean end-point verbal memory $T$-score remained in the impaired range (mean 33.9, s.d. $=12.8$ ), with $59.2 \%$ of participants scoring in this range. Mean inhibitory control $T$-scores were within normal clinical limits at baseline (mean 48.8, s.d. $=9.8$ ) and end-point (mean 51.0, s.d. $=11.0$ ), with $10.0 \%$ and $8.7 \%$ scoring within the clinically impaired range, respectively.

\section{Trajectories of cognitive change over time, and effects of primary risk factors}

Participants included in the models had on average 5.0 (s.d. $=2.6$ ) cognitive assessments with a mean of 1.3 years (s.d. $=0.5$ ) between assessments, and a mean of 6.0 years $($ s.d. $=2.3)$ total follow-up time.

\section{Verbal learning}

Verbal learning (Table 2) was characterised by an initial improvement in performance followed by a declining pattern of performance over time (quadratic time function), but this did not meet the threshold for statistical significance. Higher verbal learning scores at baseline were associated with younger age, being female, more years of education, lower total virus exposure and absence of a psychotic illness. None of the primary risk factors altered the trajectory of cognitive change over time. Addition of risk factors to the model accounted for $1.6 \%$ of the variance in verbal learning trajectories (consistent with a small to trivial effect size).

\section{Verbal memory}

Like verbal learning, an initial improvement in performance was observed. Higher verbal memory scores at baseline were associated with being female, more years of education, lower total virus exposure and the absence of a psychotic illness. A history of traumatic brain injury and alcohol dependence at baseline were associated with significantly greater decline in verbal memory over time (Table 2, Fig. 1). Addition of risk factors and their interactions to the model accounted for $5.8 \%$ of the variation in verbal memory change over time (small effect size).

\section{Inhibitory control}

The trajectory of change in inhibitory control was characterised by an initial improvement in scores followed by a pattern of significant decline over time. Of note, relatively greater decline in inhibitory control was observed for people who died during follow-up (Table 2). Higher inhibitory control scores at baseline were associated with younger age and more years of education. The risk factors and their interactions accounted for $23.0 \%$ of the pattern of change over time (medium to large effect size).

\section{Effects of secondary risk factors}

Longer duration living in an impoverished neighbourhood was associated with lower scores in verbal learning and memory at baseline, and significantly greater decline in inhibitory control over time (supplementary Table 2). Other secondary risk factors contributing to level of cognitive performance at baseline included: schizophrenia/schizoaffective disorder, HIV seropositive status, stimulant and opioid (but not cannabis) dependence, and Charlson score. Aside from duration of living in the neighbourhood, no other secondary risk factor was associated with change over time in cognitive function.

All model assumptions were deemed met. We re-ran the primary analyses excluding 27 participants who met criteria for clinical cognitive impairment (as well as eligibility for inclusion in the mixed-effects models), and the results remained unchanged. A diagnosis of clinical cognitive impairment was not associated with differential trajectories of cognitive change. Overall, our sensitivity analyses did not suggest any significant sources of bias in our data (see supplementary Table 3 and supplementary Appendix 1 for details).

\section{Cognitive function and early mortality}

As of 22 August 2018, participants were followed for a median of 6.4 years (interquartile range 3.9-8.6 years). During 2260 person-years of observation, 70 of 375 (18.7\%) participants died. Causes of death were: physical illness 30 (42.9\%), accidental overdose 24 (34.3\%), trauma 4 (5.7\%), suicide 1 (1.4\%), and unknown 11 (15.7\%). 

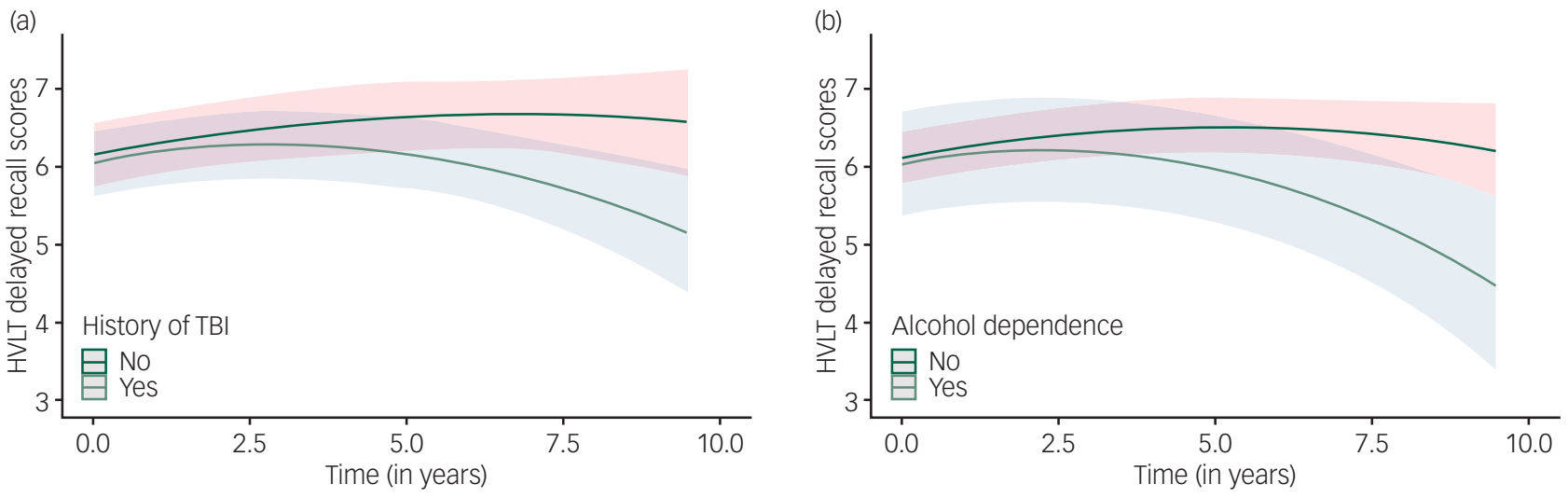

Fig. 1 Change in verbal memory over time as a function of (a) traumatic brain injury or (b) alcohol dependence.

HVLT, Hopkins Verbal Learning Test-Revised; TBI, traumatic brain injury

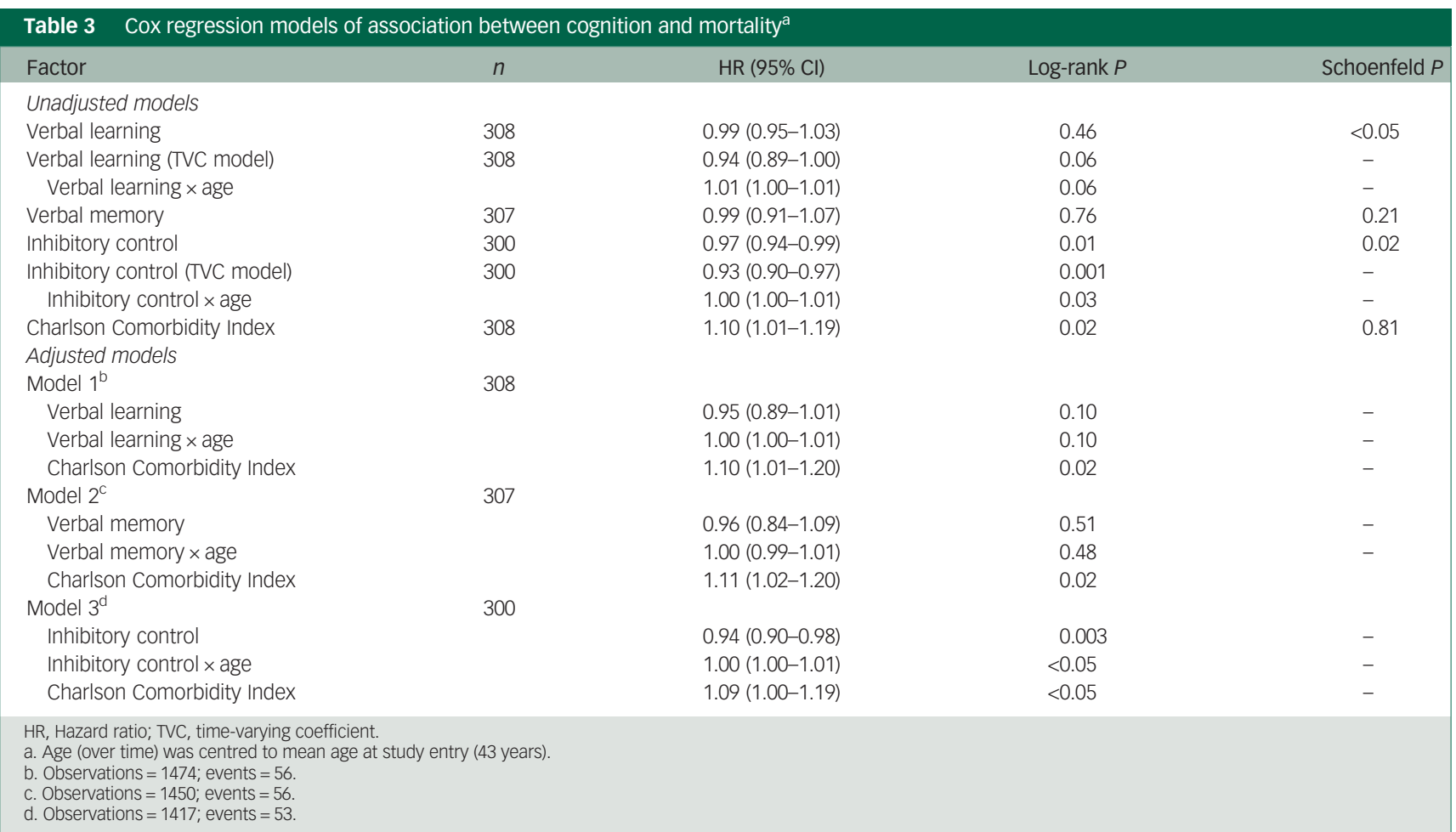

\section{Unadjusted models}

In unadjusted Cox regressions, significant Schoenfeld tests indicated that verbal learning and inhibitory control interacted with age, but there was no statistically significant effect of verbal learning over time on risk of mortality (Table 3). For inhibitory control, each additional correct response on the inhibitory control task (within a $45 \mathrm{~s}$ interval) at study entry was associated with a $6.6 \%$ lower risk of mortality, and this effect increased by $0.3 \%$ for every additional year of life. For each 1-point increase in the Charlson Comorbidity Index score at study entry, the risk of mortality was $9.9 \%$ higher, and was consistent across age. Verbal memory scores were not associated with risk of mortality.

\section{Adjusted models}

In the final model adjusted for comorbidities, inhibitory control remained a significant predictor of mortality (Table 3). Higher scores at study entry were protective, and this effect increased with age. These effects were not seen with verbal learning or memory, and were not apparent diagnostically at baseline. A diagnosis of clinical cognitive impairment at study entry was not associated with risk of mortality in those under 55 years (hazard ratio $(\mathrm{HR})=1.75, \mathrm{CI}=0.58-4.29, P=0.32$ ) or 55 years and older $(\mathrm{HR}=0.24, \mathrm{CI}=0.03-1.79, P=0.16)$. This age cut-point was suggested through inspection of the Schoenfeld residual plot (Schoenfeld $P=0.02$ ). The results were similar when demographically corrected cognitive $T$-scores were used to classify impairment ( $\leq 1.5$ s.d. below the mean).

\section{Discussion}

\section{Main findings}

Our 9-year longitudinal study of people living in a socially marginalised neighbourhood found progressive impairment in select 
cognitive domains and an association with mortality. Cognitive trajectories showed a pattern of initial improvement in performance, likely attributable to practice effects. These effects are thought to be most prominent following initial repeat testing sessions, because of familiarity with test materials and/or procedures, but diminish with additional repeat testing. ${ }^{33}$ Subsequent decline in verbal memory was most notable for individuals with a history of traumatic brain injury or alcohol dependence at baseline, controlling for the effects of sociodemographic factors, psychotic disorders and virus burden on baseline level of performance. Significant decline in inhibitory control was observed, with greater decline for those who died during follow-up and for those who spent more years living in an impoverished environment. In survival analyses, better inhibitory control predicted lower risk of mortality; this protective effect was stronger with increasing age. Risk of mortality did not differ by clinical cognitive impairment status at study entry, suggesting findings are not merely a reflection of pre-existing global cognitive deficit or dementia.

\section{Age and cognitive decline}

Patterns of cognitive decline in this comparatively young community sample appear to emerge somewhat earlier than might be expected. A longitudinal evaluation of healthy community-dwelling participants reported decline in memory and reasoning abilities beginning in the $60 \mathrm{~s}^{34}$ In a large US national sample of healthy adults, decline in immediate word list recall (verbal learning) began in the 40s, followed by decline in delayed word list recall (verbal memory) and executive functioning (reasoning, verbal fluency, working memory) beginning in the 50s and later, though these effects were reported to be very small to trivial. ${ }^{35}$ In comparison, we observed decline in verbal memory and inhibitory control (a select component of executive functioning) beginning in the latter half of the 40s. However, these comparison cohorts differ from our sample in sociodemographic composition and therefore do not provide optimal benchmarks. ${ }^{34,35}$ Although it is tempting to speculate a pattern of premature ageing, this hypothesis requires further investigation with a demographically matched control group that more closely approximates the current sample.

\section{Individual-level risk factors}

Select risk factors previously implicated in accelerated ageing had an impact on cognitive decline in this sample. Alcohol dependence was associated with greater decline in verbal memory, whereas dependence on other non-prescribed substances was not. Heavy alcohol consumption with increasing age is linked with greater impairment in learning, memory and motor functions, but not attention and executive functioning, and is considered to reflect a dual premature-accelerated model of cognitive ageing. ${ }^{36}$

Traumatic brain injury in our sample is associated with abnormalities of neural connectivity and cross-sectional cognitive impairment, ${ }^{37}$ and is now observed to be associated with steeper memory decline. A study of nearly 2.8 million people from Denmark found an increased risk of all-cause dementia in people with a history of traumatic brain injury. ${ }^{27}$ Existing evidence appears strongest for a link between severe traumatic brain injury and accelerated ageing. ${ }^{38}$ We also found that people who died during follow-up showed steeper decline in inhibitory control. This is consistent with evidence of accelerated cognitive decline several years prior to death in older adults with and without dementia. ${ }^{39}$

\section{Environmental-level risk factors}

Social and environmental deprivation may also be a major determinant of accelerated cognitive ageing. ${ }^{40}$ In the current study, longer time living within an impoverished neighbourhood was associated with greater decline in inhibitory control, possibly reflecting the cumulative effects of socioeconomic disadvantage, unsafe living conditions and social stressors. Lack of community resources for cognitive enrichment in day-to-day life may also contribute. These findings highlight the importance of considering individual-level and environment-level risk factors for cognitive decline.

\section{Factors associated with mortality}

Trajectories of cognitive functioning appear to have significant bearing on premature mortality. In a cross-sectional study of over 1100 community-dwelling older adults using a short form of the Mini-Mental Status Examination, the association between global cognition and mortality was reported to be distinct from the effects of chronic disease. ${ }^{17}$ In the present longitudinal study using more detailed neuropsychological testing, better inhibitory control was associated with a $6.6 \%$ decreased risk of mortality, and this protective effect of cognition became larger by $0.3 \%$ for every additional year of life, controlling for co-occurring chronic medical illnesses. Cognition itself may be an independent marker of health and reflective of diminished integrity in neural systems, associated with effects on brain structure and connectivity. ${ }^{10,41}$ This model is consistent with the concept of frailty, associated with decreased physiological reserve as a consequence of lifetime aggregation of effects from biological ageing or subthreshold disease-related insults. ${ }^{18}$ Cognitive dysfunction, therefore, may be an emergent clinical expression of frailty and may help to explain the stronger relationship between cognition and risk of mortality over time.

\section{Limitations}

Limitations should be considered. First, the extent of cognitive decline in the present sample was not directly compared with a healthy cohort of individuals with the same age and education, necessary for more definitive understanding of the role of premature and/or accelerated cognitive ageing. Second, the deleterious effects of alcohol and traumatic brain injury may have been underestimated because of the challenges of retrospectively assessing patterns of alcohol use, as well as severity and number of traumatic brain injuries. Additionally, we did not examine all possible cognitive domains and therefore our understanding of trajectories of change in this sample remains incomplete. We also did not examine other clinical conditions, such as mood disorders, anxiety and post-traumatic stress disorder, which are more likely to fluctuate over time and may co-vary with the level of cognitive performance at any given time point. Future studies will need to address these factors in order to provide a more complete clinical picture.

Contrary to our initial hypothesis, age, psychosis and virus exposure burden did not predict cognitive change, possibly requiring longer follow-up periods. An examination of symptoms of psychosis, rather than diagnosis, may also reveal unique covariation with cognition over time. Alternatively, for some risk factors, the association with decline may be masked by practice effects inherent in longitudinal designs. ${ }^{34}$

Lastly, the numbers of available participants limited the ability to assess possible synergistic effects of comorbidities. Nonetheless, this study has strengths in the breadth of risk factors evaluated and frequency of follow-up over 9-years. Similar risk factors are reported in homeless and marginally housed populations worldwide, ${ }^{1,3}$ and the findings may be more broadly relevant. We used well-validated neuropsychological measures that could be practically implemented in clinical services for this population, yielding unique information about cognitive status beyond what global 
screening tools can provide (for example Mini-Mental Status Examination).

\section{Implications}

Our findings of domain-specific cognitive decline and an association with mortality raise the question as to whether a dual premature-accelerated pathway of cognitive ageing is operant in homeless and precariously housed people. Alcohol use and traumatic brain injury may be modifiable risk factors, amenable to early intervention to mitigate risk for cognitive decline. Inhibitory control appears to be a protective factor; building cognitive reserve early on could buffer against decline and death. Replicating the current findings in other homeless and precariously housed samples, and across more cognitive domains, has a degree of urgency proportionate to globally increasing public health relevance.

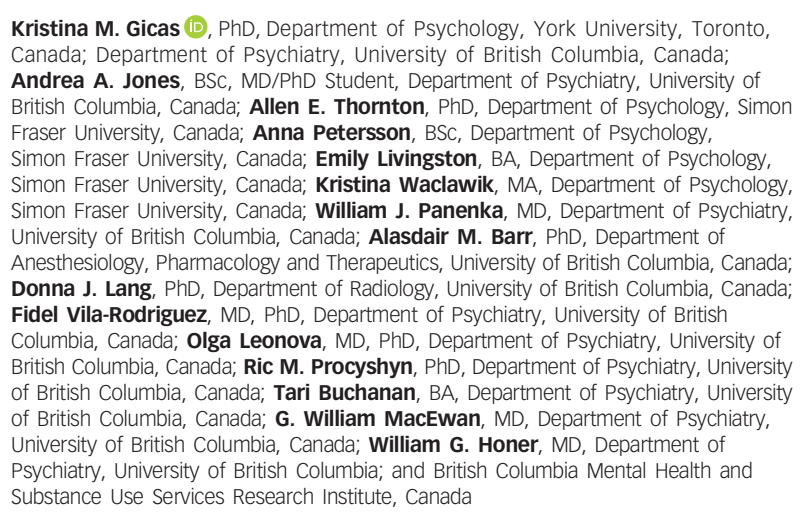

Correspondence: Kristina M. Gicas. Email: kgicas@yorku.ca

First received 29 Jul 2019, final revision 11 Jan 2020, accepted 14 Jan 2020

\section{Funding}

This study was funded by grants from the Canadian Institutes of Health Research (W.G.H., CBG101827, MOP-137103), BC Mental Health and Addictions Services (W.G.H.), and the William and Ada Isabelle Steel Fund (A.E.T.). W.G.H. was supported by the Jack Bell Chair in Schizophrenia.

\section{Acknowledgements}

We would like to thank the Hotel Study staff and volunteers for their efforts in data collection and management. We also wish to thank the study participants for their time.

\section{Data availability}

All authors have full ongoing access to the database used for carrying out this study. Data cannot be made publicly available due to possible privacy breaches and other ethical and legal obligations to the study participants. These restrictions are outlined by the University of British Columbia's Clinical Research Ethics Board and Simon Fraser University's Research Ethics Board. Inquiries regarding data can be made to the Clinical Research Ethics Board of the University of British Columbia (ethics.research.ubc.ca) and the study principal investigator (w.G.H.) atwilliam.honer@ubc.ca.

\section{Author contributions}

K.M.G., A.A.J., A.E.T. and W.G.H. were involved in formulating research questions, study design, data analysis and interpretation and writing the article. A.E.T., W.J.P., A.M.B., D.J.L., F.V.-R., R.M.P., G.W.M. and W.G.H. were involved in study conceptualisation and data acquisition and/or interpretation. A.P., E.L., K.W., O.L. and T.B. were involved in data acquisition. All authors critically revised the manuscript for intellectual content and approved the final version.

\section{Supplementary material}

Supplementary material is available online at https://doi.org/10.1192/bjo.2020.3.

\section{References}

1 Aldridge RW, Story A, Hwang SW, Nordentoft M, Luchenski SA, Hartwell G, et al. Morbidity and mortality in homeless individuals, prisoners, sex workers, and individuals with substance use disorders in high-income countries: a systematic review and meta-analysis. Lancet 2018; 391: 241-50.

2 Luchenski S, Maguire N, Aldridge RW, Hayward A, Story A, Perri P, et al. What works in inclusion health: overview of effective interventions for marginalised and excluded populations. Lancet 2018; 391: 266-80.

3 Fazel S, Geddes JR, Kushel M. The health of homeless people in high-income countries: descriptive epidemiology, health consequences, and clinical and policy recommendations. Lancet 2014; 384: 1529-40.

4 Vila-Rodriguez F, Panenka WJ, Lang DJ, Thornton AE, Vertinsky T, Wong H, et al. The Hotel study: multimorbidity in a community sample living in marginal housing. Am J Psychiatry 2013; 170: 1413-22.

5 Barbic SP, Jones AA, Woodward M, Piercy M, Mathias S, Vila-Rodriguez F, et al. Clinical and functional characteristics of young adults living in single room occupancy housing: preliminary findings from a 10 -year longitudinal study. Can J Pub Health 2018; 109: 204-14.

6 Brown RT, Kiely DK, Bharel M, Mitchell SL. Geriatric syndromes in older homeless adults. J Gen Intern Med 2012; 27: 16-22.

7 Hurstak E, Johnson JK, Tieu L, Guzman D, Ponath C, Lee CT, et al. Factors associated with cognitive impairment in a cohort of older homeless adults: results from the HOPE HOME study. Drug Alc Depend 2017; 178: 562-70.

8 Depp CA, Vella L, Orff HJ, Twamley EW. A quantitative review of cognitive functioning in homeless adults. J Nerv Ment Dis 2015; 203: 126-31.

9 Ennis N, Roy S, Topolovec-Vranic J. Memory impairment among people who are homeless: a systematic review. Memory 2015; 23: 695-713.

10 Gicas KM, Giesbrecht CJ, Panenka WJ, Lang DJ, Smith GN, Vila-Rodriguez F, et al. Structural brain markers are differentially associated with neurocognitive profiles in socially marginalized people with multimorbid illness. Neuropsychol 2017; 31: 28-43.

11 Fry CE, Langley $\mathrm{K}$, Shelton $\mathrm{KH}$. A systematic review of cognitive functioning among young people who have experienced homelessness, foster care, or poverty. Child Neuropsychol 2017; 23: 907-34.

12 Waclawik K, Jones AA, Barbic SP, Gicas KM, O'Connor TA, Smith GN, et al. Cognitive impairment in marginally housed youth: prevalence and risk factors. Front Public Health 2019; 7: 270.

13 Guggenmos M, Schmack K, Sekutowicz M, Garbusow M, Sebold M, Sommer C, et al. Quantitative neurobiological evidence for accelerated brain aging in alcohol dependence. Transl Psychiatry 2017; 7: 1279.

14 Tremblay S, De Beaumont L, Henry LC, Boulanger Y, Evans AC, Bourgouin P, et al. Sports concussions and aging: a neuroimaging investigation. Cereb Cortex 2013; 23: 1159-66.

15 Czepielewski LS, Massuda R, Panizzutti B, Grun LK, Barbé-Tuana FM, Teixeira $\mathrm{AL}$, et al. Telomere length and CCL11 levels are associated with gray matter volume and episodic memory performance in schizophrenia: evidence of pathological accelerated aging. Schizophrenia Bull 2018; 44: 158-67.

16 Pfefferbaum A, Zahr NM, Sassoon SA, Kwon D, Pohl KM, Sullivan ED. Accelerated and premature aging characterizing regional cortical volume loss in human immunodeficiency virus infection: contributions from alcohol, substance use, and hepatitis C coinfection. Biol Psychiatry Cogn Neurosci Neuroimaging 2018; 3: 844-59.

17 Schultz-Larsen K, Rahmanfard N, Kreiner S, Avlund K, Holst C. Cognitive impairment as assessed by a short form of MMSE was predictive of mortality. $J$ Clin Epidemiol 2008; 61: 1227-33.

18 Fried LP, Ferrucci L, Darer J, Williamson JD, Anderson G. Untangling the concepts of disability, frailty, and comorbidity: implications for improved targeting and care. J Gerontol A Biol Sci Med Sci 2004; 59: 255-63.

19 Honer WG, Cervantes-Larios A, Jones AA, Vila-Rodriguez F, Montaner JS, Tran H et al. The Hotel study-clinical and health service effectiveness in a cohort of homeless or marginally housed persons. Can J Psychiatry 2017; 62: 482-92.

20 Krausz M, Jang K. Lessons from the creation of Canada's poorest postal code. Lancet Psychiatry 2015; 2: e5.

21 Gaetz S, Barr C, Friesen A, Harris B, Hill C, Kovacs-Burns K, et al. Canadian Definition of Homelessness. Canadian Observatory on Homelessness Press, 2012.

22 Benedict RHB, Schretlen D, Groninger L, Brandt J. Hopkins Verbal Learning Test Revised: normative data and analysis of inter-form and test-retest reliability. Clinical Neuropsychol 1998; 12: 43-55.

23 Sheehan DV, Lecrubier $Y$, Sheehan $\mathrm{KH}$, Amorim $\mathrm{P}$, Janavs J, Weiller $\mathrm{E}$, et al. The mini-international neuropsychiatric interview (M.I.N.I.): the development and validation of a structured diagnostic psychiatric interview for DSM-IV and ICD-10. J Clin Psychiatry 1998; 59(Suppl 20): 22-33.

24 American Psychiatric Association. Diagnostic and Statistical Manual of Mental Disorders (4th edn) (DSM-IV). American Psychiatric Association, 1994.

25 Lieberman JA, First MB. Psychotic disorders. N Engl J Med 2018; 379: 270-80. 
26 Petersen RC. Mild cognitive impairment as a diagnostic entity. J Intern Med 2004; 256: 183-94.

27 Fann JR, Ribe AR, Pedersen HS, Fenger-Grøn M, Christensen J, Benros ME, et al. Long-term risk of dementia among people with traumatic brain injury in Denmark: a population-based observational cohort study. Lancet Psychiatry 2018; 5: 424-31.

28 Noppert GA, Aiello AE, O'Rand AM, Cohen HJ. Investigating pathogen burden in relation to a cumulative deficits index in a representative sample of US adults. Epidemiol Infect 2018; 146: 1968-76.

29 Charlson ME, Pompei P, Ales KL, Mackenzie CR. A new method of classifying prognostic comorbidity in longitudinal studies: development and validation. J Chronic Dis 1987; 40: 373-83.

30 Kwok O-M, Underhill AT, Berry JW, Luo W, Elliott TR, Yoon M. Analyzing longitudinal data with multilevel models: an example with individuals living with lower extremity intra-articular fractures. Rehabil Psychol 2008; 53: 370-86.

31 Bates $D$, Mächler M, Bolker B, Walker SC. Fitting linear mixed-effects models using Ime4. J Stat Software 2015; 67: 1-48.

32 Therneau TM, Grambsch PM. Modeling Survival Data: Extending the Cox Model. Springer, 2000.

33 Wilson RS, Capuano AW, Yu L, Yang J, Kim N, Leurgans $S E$, et al. Neurodegenerative disease and cognitive retest learning. Neurobiol Aging 2018; 66: 122-30

34 Salthouse TA. Trajectories of normal cognitive aging. Psychol Aging 2019; 34: $17-24$
35 Hughes ML, Agrigoroaei S, Jeon M, Bruzzese M, Lachman ME. Change in cognitive performance from midlife into old age: findings from the Midlife in the United States (MIDUS) study. J Int Neuropsychol Soc 2018; 24: 805-20.

36 Woods AJ, Porges EC, Bryant VE, Seider T, Gongvatana A, Kahler CW, et al. Current heavy alcohol consumption is associated with greater cognitive impairment in older adults. Alcohol Clin Exp Res 2016; 40: 2435-44.

37 Schmitt T, Thornton AE, Rawtaer I, Barr AM, Gicas KM, Lang DJ, et al. Traumatic brain injury in a community-based cohort of homeless and vulnerably housed individuals. J Neurotrauma 2017; 34: 3301-10.

38 Wood RL. Accelerated cognitive aging following severe traumatic brain injury: a review. Brain Inj 2017; 31: 1270-8.

39 Karr JE, Graham RB, Hofer SM, Muniz-Terrera G. When does cognitive decline begin? A systematic review of change point studies on accelerated decline in cognitive and neurological outcomes preceding mild cognitive impairment, dementia, and death. Psychol Aging 2018; 33: 195-18.

40 Clarke PJ, Weuve J, Barnes L, Evans DA, Mendes de Leon CF. Cognitive decline and the neighborhood environment. Ann Epidemiol 2015; 25: 849-54.

41 Gicas KM, Jones AA, Panenka WJ, Giesbrecht C, Lang DJ, Vila-Rodriguez F, et al. Cognitive profiles and associated structural brain networks in a multimorbid sample of marginalized adults. PLOS ONE 2019; 14: e0218201. 